\title{
Hypothalamic Amenorrhea: Causes, Complications, \& Controversies
}

\author{
Taylor Collins $^{\mathrm{a}}$ and Krista L. Rompolski ${ }^{\mathrm{a}}$
}

Hypothalamic amenorrhea (HA) is considered a reversible condition characterized by the absence of menses for 3 months or more, due to suppressed secretions of gonadotropin releasing hormone affecting the entire hypothalamic-pituitary-ovarian axis. HA can be triggered by excessive stress, weight loss or excessive exercise, however, the etiology is still largely unknown. Serious, long-term complications include severe hypoestrogenism and infertility, in addition to a variety of hormonal aberrations. Hypoestrogenism also leads to diminished bone health, cardiovascular problems, and mood changes that lead to a higher prevalence of depression and anxiety. It is important that HA is diagnosed in a timely manner in order to begin therapeutic strategies that aim to resume menses and return to normal levels of circulating reproductive hormones. When attempts to resume menstruation naturally through lifestyle changes are unsuccessful, other pharmaceutical options are available. Treatment options range from estrogen-replacement therapy to the administration of gonadotropin releasing hormone, depending on the reproductive goals of the woman. More research is needed on novel treatments in order to determine the most effective standard of care.

Keywords: Hypothalamic amenorrhea; Gonadotropin-releasing hormone; Hypoestrogenism; Leptin; Female Athlete Triad

\section{Introduction}

Hypothalamic amenorrhea (HA) is a form of secondary amenorrhea that affects 3-5\% of adult women (Meczekalski, Podfigurna-Stopa, Warenik-Szymankiewicz, \& Genazzani, 2008). Unlike primary amenorrhea, which is the absence of menarche altogether, this absence from normal menstruation for 3 months or more is due to a suppression of the hypothalamic-pituitary-ovarian axis resulting in impaired gonadotropin releasing hormone $(\mathrm{GnRH})$ and gonadotropin secretion (Berga et al., 1989). Also commonly referred to as functional hypothalamic amenorrhea (FHA), HA is characterized by three main types according to the physiologic or psychological trigger. These include excessive stress, weight loss or excessive exercise, and in many cases, the patient will present with all three factors (Meczekalski et al., 2008). FHA is a diagnosis of exclusion with no evidence of organic disease, endocrine disease, and central nervous system disease (Genazzani, Ricchieri, Lanzoni, Strucchi, \& Jasonni, 2006). Proper and careful diagnosis is crucial for women affected with FHA in order to prevent short- and longterm medical consequences resulting from the altered hormonal profile associated with amenorrhea (Meczekalski et al., 2008).

Diminished function of the hypothalamic-pituitaryovarian axis may cause a multitude of complications, including diminished reproductive potential, reduced bone strength, metabolic disorders, and altered mental health (Meczekalski et al., 2008). In terms of reproductive health, anovulation and infertility are two of the major concerns for women with FHA (Hind, 2008). Without GnRH secretion, luteinizing hormone ( $\mathrm{LH}$ ) and follicle-stimulating hormone (FSH) secretion are compromised, resulting in anovulation (Meczekalski et al., 2008). In addition, hypoestrogenism is caused by the impaired gonadotropin secretion, which has a seriously negative impact on a variety of aspects of female health (Meczekalski et al., 2008). Especially in patients suffering from hypoestrogenism for more than several months, reduced serum estradiol levels may negatively affect peak bone mass, increasing the risk of pathological fractures (Genazzani, 2005). Moreover, there may be metabolic consequences, including suppressed triiodothyronine $\left(\mathrm{T}_{3}\right)$, leptin, insulin, and insulin-like growth factor-1 (IGF-1), which also play a role in reduced bone mass density due to their influence on bone turnover (De Souza \& Williams, 2005). Estrogen also has a positive effect on the cardiovascular system, and hypoestrogenism has been shown to lead to endothelial dysfunction, decreased nitric oxide bioavailability, and activation of the renin-angiotensin system (Mendelsohn \& Karas, 1999). Lastly, mental health is also negatively affected by hypoestrogenism, with women experiencing higher prevalence of depression and anxiety disorders (Lawson et al., 2009). Mood changes are associated with the hormonal aberrations caused by the hypoestrogenic conditions and over activity of the hypothalamic-pituitaryadrenal axis, although there are very few studies demonstrating the relationship (Giles \& Berga, 1993; Meczekalski, Katulski, Czyzyk, Podfigurna-Stopa, \& Maciejewski-Jeske, 2014).

FHA is a very complex syndrome with much of its pathophysiology still unknown. Because there is more than one factor that often leads to amenorrhea, more than one therapeutic approach is often needed. The purpose of this literature review is to examine the implications hypothalamic amenorrhea has on women's health and the various methods of treatments that are available. The known pathophysiology, diagnosis, theoretical causes, consequences, and possible treatments of HA will be reviewed. Different therapeutic approaches will be explored in order to determine the best mode of care for specific populations of women, depending on their lifestyle, reproductive goals and severity of physiologic consequences. In addition, this review aims to clarify controversies in HA diagnosis and treatment and identify areas in need of future research. 


\section{What is Hypothalamic Amenorrhea?}

Functional hypothalamic amenorrhea (FHA) is a condition characterized by dysfunction of the hypothalamicpituitary-ovarian axis causing anovulation and the absence of menses without the presence of other organic disease (Meczekalski et al., 2008).

To begin, the exact mechanisms explaining the pathophysiology of FHA are intricate and unclear (Marshall, 1994). In order to understand the variations that could cause FHA to occur, it is important to understand the physiology of a normal menstrual cycle. GnRH is produced and released from the hypothalamus into the hypophyseal portal system where it is transported to the anterior pituitary in pulses, or short bursts of releasing hormone, every 90 to 120 minutes (Snyder, Reitano, \& Utiger, 1975). Once in the anterior pituitary, GnRH stimulates the synthesis and secretion of luteinizing hormone (LH) and follicle stimulating hormone (FSH), which are also secreted in a pulsatile manner following the $\mathrm{GnRH}$ pulse. In a normal menstruating female, FSH levels rise during the follicular phase of menarche to stimulate the growth of an ovarian follicle and estrogen production. Once estrogen reaches a threshold level, a GnRH triggers the rapid release of LH, known as the LH surge and ovulation occurs soon after. After the follicle is released, the corpus luteum forms, which produces and releases progesterone. If implantation does not occur, the corpus luteum degenerates, progesterone levels fall, and the endometrial lining is sloughed during menses (Golden \& Shenker, 1994).

Women affected with FHA have a spectrum of hypothalamic-pituitary disturbances that stem from the diminished secretions of $\mathrm{GnRH}$ from the hypothalamus (Meczekalski et al., 2008). GnRH pulses are determined by serum levels of sex hormones, and are also regulated by numerous neurotransmitters and neuropeptides, including dopamine, endogenous opioids, norepinephrine, gamma amino butyric acid (GABA), corticotropin-releasing hormone $(\mathrm{CRH})$, kisspeptin, leptin, and ghrelin (Golden \& Shenker, 1994; Meczekalski et al., 2008). When the body detects severe stressors, whether metabolic, physical, or psychological stress, it responds with adaptive mechanisms that trigger various physiological pathways for survival (Genazzani, 2005). These mechanisms cause numerous endocrine and neuroendocrine abnormalities that disable normal functioning of the reproductive axis as seen in women with FHA.

In FHA, the entire hypothalamic-pituitary-gonadal axis is suppressed, ultimately resulting in reduced or blocked ovarian function (Bomba et al., 2007). The main endocrine abnormalities resulting from the diminished $\mathrm{GnRH}$ secretions are low LH plasma levels and hypoestrogenism (Genazzani et al., 1990). Without normal LH pulsatility, ovulation does not occur and the woman becomes infertile. This is a serious consequence of FHA for women who are wishing to conceive (Genazzani, 2005). Hypoestrogenism, on the other hand, is a serious consequence of FHA for all women due to the negative impact low estradiol levels have on numerous aspects of women's health (Meczekalski et al., 2008).

The hypothalamic-pituitary-adrenal axis can become overactive under stressful conditions resulting in an increase of corticotropin releasing hormone $(\mathrm{CRH})$ in the central nervous system, which may increase secretions of adrenocorticotropin hormone (ACTH) from the pituitary and cortisol from the adrenal glands (Berga, Daniels, \& Giles, 1997). Elevated levels of CRH also cause an increase in endogenous opioids, especially beta-endorphin, which is directly linked to reduced GnRH and gonadotropin secretion (Genazzani, 2005)

Alterations in the hypothalamic-pituitary-thyroid axis are also observed in women with FHA. Low-to-normal levels of thyroid stimulating hormone (TSH), low levels of triiodothyronine, and increased levels of reverse triiodothyronine have been seen (Berga et al., 1997). Other aberrations include significantly lower concentrations of leptin and elevated concentrations of ghrelin (Bomba et al.; De Souza, Leidy, O'Donnell, Lasley, \& Williams, 2004). Leptin, a hormone secreted by adipose tissue, alerts the body of its nutritional status and energy balance. By directly decreasing GnRH secretions and indirectly increasing CRHACTH-cortisol secretions, leptin deficiency plays a crucial role in the hypothalamic disturbances with FHA (Macut, Micic, Pralong, Bischof, \& Campana, 1998). Ghrelin, on the other hand, is a neuropeptide produced in the stomach and intestinal tract that is responsible for stimulating hunger, food intake, and energy homeostasis (De Souza, Leidy, O'Donnell, Lasley, \& Williams, 2004). In a recent study performed on ovariectomized rats, researchers demonstrated the inhibitory effect ghrelin has on LH pulse frequency (Furuta, Funabashi, \& Kimura, 2001). Because those affected by FHA are characterized by elevated levels of ghrelin, it is likely ghrelin is also a mediator of the diminished gonadotropin secretions at the hypothalamic level (De Souza et al., 2004).

Due to the largely unknown etiology of FHA, it is a diagnosis of exclusion. Other forms of primary and secondary amenorrhea should be distinguished from FHA, including genetic causes such as Kallman syndrome and Prader-Willi Syndrome (Caronia et al.). In order to exclude organic diseases of the hypothalamic area, including but not limited to neoplasms, sarcoidosis, tuberculosis, and parasitoids, magnetic resonance imaging (MRI) may be needed (Meczekalski et al., 2014). In addition, endocrine diseases and metabolic disorders such as diabetes and Polycystic Ovary Syndrome (PCOS) need to be ruled out. Upon an initial visitation, the patient's clinical history regarding menarche, menstrual cyclicity, time and expression of amenorrhea should be questioned, as well as their psychiatric history involving depressive states and mood disorders (Genazzani, Ricchieri, Lanzoni, Strucchi \& Jasonni, 2006). It is important to gain an understanding of the patient's stressors, including stress caused by loss, family or work situations, weight loss or eating disorders, or intense physical training (Genazzani et al., 2006). If the patient is taking any medications that could cause amenorrhea, they need to be ruled out as well, specifically antipsychotics and contraceptive agents, due to their effects on the anterior pituitary gland which may cause fluctuations in the serum levels of estradiol (WikstenAlmstromer, Hirschberg, \& Hagenfeldt, 2007).

Upon physical examination of the patient, signs of hyperandrogenism should be ruled out. With a bimanual pelvic examination, the presence of a foreign body or uterine mass can be excluded, and in estrogen-deficient women, reddened, thin mucosal lining may be observed (Gordon, 2010). Laboratory blood tests can rule out a number of other 
conditions and chronic diseases. Basic tests to rule out pregnancy, thyroid disease, and hyperprolactinemia are also used (Golden \& Carlson, 2008). In addition, a full assessment of the gonadotropins is necessary, particularly to rule out ovarian insufficiency (Gordon, 2010; Golden \& Carlson, 2008). Women with FHA are characterized by having low-tonormal levels of LH and FSH while the gonadotropin response to GnRH stimulation still exists (Gordon, 2010). The key diagnostic tool used to identify hypogonadotropic hypogonadism is a GnRH stimulation test, which will show a positive gonadotropin response to the exogenous $\mathrm{GnRH}$ in the case of FHA (Meczekalski et al., 2008).

\section{Causes \& Complications}

There are three types of functional hypothalamic amenorrhea classified according to its physiological or psychological trigger. These triggers include weight loss, excessive exercise, and excessive stress (Meczekalski et al., 2008). In many cases the patient will present with more than one cause, if not all three, which makes it difficult to distinguish exactly what mechanism may be the driving force. Overall, it is the state of chronic energy deficiency that can occur with both weight-loss and exercise-induced HA that suppresses the hypothalamic-pituitary-ovarian axis. Similarly, the overlap between physical and psychological stressors also makes the origin of onset hard to differentiate. Both triggers elicit adaptive mechanisms in the body to redirect energy and resources away from functions non-essential for survival such as reproduction, and toward more crucial functions, such as the cardiovascular and central nervous systems (Liu \& Bill, 2008).

Prolonged restrictive eating patterns greatly diminish GnRH release from the hypothalamus (Golden \& Shenker, 1994). Regardless of whether the woman is underweight or of normal weight, inadequate caloric intake causes a negative energy balance, interrupting the body's homeostasis. There is the ongoing debate on whether there is a critical level of body fat that must be met for menses, however, more populationbased studies must be done before coming to a conclusion. One recent study done by Golden, Jacobson, Sterling and Hertz examined 56 females with FHA also being treated for anorexia nervosa, in order to determine goal weight for the onset of menses. Of the 56 women, 36 resumed menses and 20 remained amenorrheic within the first year of follow-up. There was no significant differences in weight, percent body fat, or BMI between the two groups, but fifty percent of the women who did resume menses did so once they reached a BMI between the $14^{\text {th }}$ and $39^{\text {th }}$ percentile (Golden, Jacobson, Sterling \& Hertz, 2008). Altogether, women who do not meet their energy needs over an extended period of time will lose their reproductive functions regardless of their body weight (Nattiv et al., 2007).

In addition, lower-than-normal circulating levels of leptin reflecting a state of energy deficiency could be the acute cause of amenorrhea even before any weight loss occurs (Golden et al., 2008). Leptin receptors located in the areas of the hypothalamus associated with eating behaviors, energy balance, and reproduction regulation suggest the role that leptin plays on the reproductive system. When the receptors sense low levels of leptin, the hypothalamus responds by inhibiting $\mathrm{GnRH}$ release resulting in menstrual dysfunction
(Macut et al., 1998). In a study aimed to determine the correlations between leptin, body weight, and BMI in women with FHA, eighty-eight women with FHA were compared to sixty-five controls with comparable age, mean body weight, and mean BMI. There was a strong linear positive correlation between plasma leptin levels and body weight, and plasma leptin levels and BMI, however, the study showed women with FHA had significantly lower levels of leptin, even in patients with normal body weight and BMI (Andrico et al., 2002). Another study done by Kolaczynski, Ohannesian, Considine, Marco, and Caro demonstrated the effect of fasting on leptin levels, dramatically decreasing leptin plasma concentrations, as well as the effect of one day of overfeeding, which does not change body weight, increasing serum leptin by $40 \%$ (Kolaczynski, Ohannesian, Considine, Marco, \& Caro, 1996). This shows the importance of maintaining a proper energy balance with adequate dietary intake and nutrition, due to the impact energy deficiency has on the reproductive axis.

Exercise-induced amenorrhea is caused by an excessive use of energies, usually due to a combination of reduced food intake and intense training (Bonen, 1994). On the other hand, long-term prospective studies have shown decreased LH pulsatility and anovulation due to increasing exercise expenditure alone (Bullen et al., 1985). According to most of the research criteria, excessive exercise is defined as greater than 10 hours of any type of exercise per week, or running more than 10 miles per week (Berga et al., 2003; Michopolous, Mancini, Loucks \& Berga, 2013). Especially seen in lean-sport athletes, such as dancers, runners, and swimmers, the fat to muscle mass ratio is significantly reduced and these athletes may be consuming too few calories to keep up with their energy needs (Nattiv et al., 2007). The female athlete triad is a psychological model proposed in 1992, which describes the relationship between low energy availability with or without an eating disorder, functional hypothalamic amenorrhea, and osteoporosis. Low energy availability, defined as energy intake minus energy expenditure, is the chief cause of reproductive dysfunction and poor skeletal health in the Triad. Females who are at greatest risk include those who restrict dietary intake, limit the types of food they consume, and exercise for extended periods of time (Nattiv et al., 2007). Recent studies have demonstrated the significance of dietary intake over exercise training as the primary cause of $\mathrm{GnRH}$ suppression and diminished LH pulsatility, known as the "energy-availability hypothesis" (Loucks, Verdun \& Heath, 1998). Compared to the "exercise-stress hypothesis" which focuses on exercise training as a chronic stressor that activates the HPA axis, new evidence supporting the energy hypothesis suggests dietary intake inadequate for energy costs is the main facilitator (Loucks, Verdun \& Heath, 1998). More research needs to be conducted on both hypotheses in order to determine the most efficient way to reverse FHA by either increasing energy intake or moderating their exercise regime.

Excessive psychosocial stress causes hormonal and neuroendocrine changes in order to allow the body to adapt to the changing internal environment. Activation of the hypothalamic-pituitary-adrenal (HPA) axis results in increased secretion of corticotropin-releasing hormone (CRH) and sympathetic nervous system response, releasing epinephrine and norepinephrine into the bloodstream, which 
increases pulse, blood pressure and respiratory rate. CRH release activates the endogenous opioid activity, betaendorphins in particular. As described in the previous section, both CRH and beta- endorphin directly inhibit GnRH pulsatility (Genazzani, 2005). Glucocorticoids are also released under stress, which have a suppressive effect on gonadotropin secretion and sex steroid biosynthesis, namely estrogen. In addition to psychological stressors inhibiting reproductive function directly, intense emotional events can have a negative impact on food intake, leading back to amenorrhea caused by chronic energy insufficiency (Cameron, Helmreich, \& Schreihofer, 1993). Other psychiatric affective disorders linked with women with FHA are worth noting. Depressive disorders, most commonly lasting two years or more, and generalized anxiety disorder are the most common among women affected by disruption of the hypothalamus-pituitary-ovarian axis (Dundon, Rellini, Tonani, Santamaria, \& Nappi, 2010; Facchinetti, Fava, Fioroni, Genazzani, \& Genazzani, 1993). However, there are very few studies on this subject, and further studies need to be completed to decipher the temporal relationship between FHA and depression and anxiety.

Regardless of the type of stressor, metabolic, physical, or psychological, functional hypothalamic amenorrhea results in a number of complications for a woman's health. Many of the implications of FHA in women are consequential to the profound hypoestrogenism, such as reduced bone mineral density, impaired cardiovascular function, and mood disorders. However, for those women wishing to conceive, infertility is a chief concern. Reproductive potential is greatly diminished, if not completely compromised, by chronic amenorrhea (Hind, 2008). Reduced function of the hypothalamus-pituitary-ovarian axis ultimately leads to reduced ovarian function, hypoestrogenism, and anovulation since LH pulse amplitude is significantly decreased (Genazzani et al., 1990). Evidently, women who are not ovulating cannot become spontaneously pregnant. Fortunately FHA is considered reversible with proper nutritional intake, lifestyle changes or possible pharmaceutical treatments, and those who recover may become pregnant after resuming menses.

Hypoestrogenism has a severe impact on the risk of bone loss. Estrogen is a regulator of skeletal homeostasis, and lack of estrogen has been shown to increase bone turnover and prevent women from achieving peak bone mass (PBM) before the age of 30 (Meczekalski, Podfigurna-Stopa \& Genazzani, 2010). Estrogens stimulate bone formation, as well as, suppress bone resorption at the cellular level. Normal serum estradiol levels are $40-50 \mathrm{pg} / \mathrm{mL}$ in women showing positive bone metabolism. According to most studies, women with FHA have serum estradiol levels below $20 \mathrm{pg} / \mathrm{mL}$, significantly less than the normal amount required for proper bone health (De Souza \& Williams, 2005; Shen, Xu \& Lin, 2013). Other factors that can negatively impact bone health and are also related to those with FHA include improper diet with low calcium and vitamin D intake. Metabolic factors relating to caloric restriction and energy deficiency seen in those with disordered eating and excessive exercise have been shown to reduce peak bone mass even in athletes who are eumenorrheic, or have normal menstruation (De Souza \& Williams, 2005).
Estrogen also has a regulatory effect on the cardiovascular system, specifically vascular function of the coronary and peripheral vessels, which contain estrogen receptors (Mendelsohn \& Karas, 1994). Hypoestrogenism has been associated with decreased bioavailability of nitric oxide, endothelial dysfunction, activation of the renin-angiotensin system, and accelerated progression of atherosclerosis, possibly due to the changes just previously mentioned (Kaplan \& Manuck, 2008). There are a number of factors that are associated with estrogen deficiency and impaired cardiovascular function, but the long-term consequences of FHA on the cardiovascular system is understudied and requires an understanding of the related complex physiological pathways (Meczekalski et al., 2014).

Women with FHA who are hypoestrogenic have neuroendocrine consequences when regulating a stress response that result in certain mood disorders and dysfunctional attitudes (Berga et al., 2003). Along with hypoestrogenism, hypercortisolemia is also one of the features associated with FHA, and there is positive correlation between cortisol levels and the Hamilton Rating Scale for Depression and Anxiety (Lawson et al., 2009). As described earlier, depressive and anxiety disorders are the most commonly documented mood disorders in a person affected with FHA. Depression is also linked with sexual dysfunction, and one study demonstrated significantly lower scores on overall sexual functioning in women with FHA (Berga et al., 2003). Characteristic attitudes associated with these endocrine adaptations include higher levels of perfectionism, higher need for social approval, and altered eating behaviors, which can affect women psychosocially (Berga et al., 2003; Marcus, Loucks \& Berga, 2001). Cognitive behavioral therapies aimed at resuming menses with psychosocial approaches will be discussed in the following section.

\section{Treatment Options}

Because the etiology of hypothalamic amenorrhea is not clearly understood, there are a variety of therapeutic approaches that aim to regain normal functioning of the reproductive system. Attempts to restore menstruation naturally without the administration of hormones and medications include weight gain, rest from exercise, and psychological therapy (Berga et al., 2003; Kopp-Woodroffe, Manore, Dueck, Skinner, \& Matt, 1999; Nattiv et al., 2007). If the chief cause of menstrual dysfunction is excessive weight loss or chronic energy deficiency, simply reversing this with weight gain and less restrictive eating patterns can restore normal functioning (Kopp-Woodroffe et al., 1999). Similarly with excessive exercise or intense athletic training, taking a rest from exercise or decreasing the number of hours spent exercising each week will typically allow menses to resume (Kopp-Woodroffe et al., 1999). In a study completed by Kopp-Woodroffe, Manore, Dueck, Skinner, and Matt at Arizona State University, four amenorrheic athletes, categorized as four separate case studies, participated in a training intervention program which monitored their eating patterns and provided a daily nutritional supplement containing $17 \mathrm{~g}$ of protein and $6 \mathrm{~g}$ of fat (Kopp-Woodroffe et al., 1999). Energy expenditure was also monitored and the athletes were required to take one day of rest per week. After 20 weeks of intervention, all four participants gained 1 
kilogram of weight, and three of the four athletes resumed menses either during or shortly following the program (KoppWoodroffe et al., 1999). Due to the design of the study, it is unclear whether the energy intake or energy expenditure was the exact cause of resumption of mense; however, both factors contribute the overall body energy balance, which is shown to play a major role in normal reproductive functioning. Casecontrol studies with larger sample sizes are needed in this area to determine whether intervention programs are effective in restoring menstruation.

In spite of the potential success of treatment for FHA, the feasibility of changing behaviors in female athletes who participate in sports that promote leanness for optimal performance is challenging. Even women who are not athletes may have a difficult time changing their long-standing eating patterns or taking a rest from exercise. In addition to balancing energy intake and expenditure, behavioral modifications may be necessary, especially for those who have trouble with body image, eating attitudes, social approval, and overall stressful events (Berga et al., 2003).

When the chief cause of hypothalamic-pituitary-ovarian axis suppression is thought to be excessive stress, therapeutic approaches to minimize the activation of the hypothalamicpituitary-adrenal axis are explored (Berga et al., 2003). Cognitive behavioral therapy (CBT) has been shown to alter problematic attitudes that have been associated with women with FHA, including dieting, fear of weight gain, insecurity, inadequacy, and higher levels of depressive symptoms (Marcus et al., 2001). In a randomized control trial involving 16 women with FHA, a 20-week intervention using CBT aimed at developing healthy eating patterns, promoting better life coping skills, and problem-solving skills in order to minimize relapse into previous maladaptive patterns. Of the eight women randomized into the CBT arm, six became ovulatory and resumed menses, whereas one woman from the observational group became ovulatory (Berga et al., 2003). In a subsequent randomized control trial using CBT to measure neuroendocrine recovery, serum levels of cortisol, TSH, $\mathrm{T}_{3}$, $\mathrm{T}_{4}$, and leptin were measured in 17 women with FHA. After 20 weeks of CBT targeting the same problematic attitudes, only the women in the CBT arm showed significantly reduced cortisol levels, as well as increased leptin and TSH levels. $\mathrm{T}_{3}$ and $\mathrm{T}_{4}$ levels remained unchanged in both groups, in addition to unchanged BMI (Michopoulos, Mancini, Loucks, \& Berga, 2013).

Hypnotherapy has also been explored in an observational study involving 12 women experiencing stress-induced FHA. Women were followed for 12 weeks after one session of hypnotherapy, which lasted 45-70 minutes. The hypnotherapy used words and suggestions intended to arouse the patient with feelings of well-being and comfort depending on each individual's situational stressors. After one session, nine of the 12 women reported vaginal bleeding within the 12-week follow-up period, including one who got pregnant after the first flow and five who had regular cycle lengths, compared to three who menstruated once within the given timeframe (Tschugguel \& Berga, 2003). Additional studies measuring the efficacy of hypnotherapy are needed to confirm this phase 1 observational study, however, hypnotherapy may be a viable option for those who are interested and believe in this type of therapy.
Altogether, a multidisciplinary approach is recommended based on clinical experience. Especially due to the number of possible causes to the onset of menstrual dysfunction, it is beneficial to seek care from not only a primary care physician and reproductive endocrinologist, but a nutritionist and psychotherapist, as well. There is little data to support the long-term efficacy of this type of treatment strategy, however, women who have taken a more holistic approach to address both metabolic and psychosocial stressors have had more success in reversing amenorrhea and anovulation, than women who focus on exercise or diet alone (Berga et al., 2003; Michopoulos et al., 2013).

When an attempt to naturally restore reproductive function is unsuccessful, or is not feasible for a particular lifestyle choice, there are a number of other pharmaceutical methods to facilitate the reversal process. Currently, the treatment of choice for women who wish to conceive by resuming ovulation is the administration of pulsatile $\mathrm{GnRH}$ or injectable gonadotropins. Pulsatile GnRH can be administered through an IV catheter to mimic the natural pulse frequencies in a normal ovulating woman. This will then stimulate the release of gonadotropins ( $\mathrm{LH}$ and FSH), which will resume follicular maturation ovulation in most cases. On the other hand, injectable gonadotropins can be administered daily to directly act on the ovaries to prompt follicular maturation and ovulation in that manner. In one study done at Massachusetts General Hospital by Martin, Hall, Adams, and Crowley, which excluded women with a history of eating disorders or excessive exercise, researchers compared the efficacy of pulsatile GnRH and gonadotropin administration in 41 women with FHA. There was a $97 \%$ ovulatory rate for those receiving exogenous gonadotropins, and $93 \%$ ovulatory rate for those receiving pulsatile $\mathrm{GnRH}$, only. There was no significant difference in conception rates; however, there was a significantly higher rate of multiple gestations with triplets or more in the women who received gonadotropins directly. This leads to more risky pregnancies and higher chance of spontaneous abortions. There were also women who had to discontinue treatment in the gonadotropin group due to multiple follicular development and high serum estradiol levels (Martin, Hall, Adams \& Crowley, 1993). Overall, the success rates of those receiving exogenous gonadotropins versus pulsatile $\mathrm{GnRH}$ are comparable, but using pulsatile $\mathrm{GnRH}$ seems to be the safer alternative by maintaining the natural pituitary-ovarian negative feedback mechanisms that only allow the maturation of one follicle and sense circulating levels of estradiol (Martin et al., 1993).

Another plausible method for restoring ovulation by stimulating LH pulsatility is the administration of kisspeptin, a hypothalamic neuropeptide recently identified as a factor in the GnRH pulse generator (Dhillo et. al., 2007). It has been shown that single injections of kisspeptin-54 stimulated the release of gonadotropins in healthy males and females (Dhillo et al., 2007) Because of these findings, recent studies have been done to evaluate the therapeutic efficacy of kisspeptin in women with FHA. Initial research demonstrated desensitization in gonadotropin responses to exogenous kisspeptin injected daily, causing a progressive reduction in LH responses after injections and virtually no FSH response after the third day (Jayasena et al., 2010). Subsequently, twice-weekly injections of kisspeptin-54 were tested in women with FHA, which stimulated the release of 
gonadotropins, but no recovery in menstruation was observed (Jayasena et al., 2014). The same researchers then hypothesized that a dose-dependent therapeutic window must exist for kisspeptin to restore GnRH and LH pulsatility. In the study done by Jayasena et al, containing five women with FHA, the women were injected with a different dose of kisspeptin-54 for 8 hours once a week. The first two lowest doses showed no change in LH secretion compared to baseline values, however there was a 4-fold increase in the mean pulsatile LH secretion at the next highest dose of 0.10 $\mathrm{nmol} / \mathrm{kg} /$ hour. The highest dose $(1.00 \mathrm{nmol} / \mathrm{kg} / \mathrm{hour})$ increased mean serum LH levels dramatically in the first 5 hours of infusion, but then decreased LH levels to $50 \%$ lower than peak values, most likely caused by the desensitization (Jayasena et al., 2014). No other adverse effects have been reported with the administration of kisspeptin (Dhillo et al., 2007; Jayasena et al., 2010; Jayasena et al., 2014). Further studies are needed to compare the efficiency of kisspeptin-54 versus pulsatile GnRH administration, before suggesting kisspeptin-based therapies in women with FHA wishing to conceive.

Leptin, fully discussed as a possible root cause of the diminished GnRH secretions, can also be administered to restore the pituitary-ovarian axis. Human recombinant leptin, or metreleptin, has been shown to resume ovulation and menses, and increase levels of LH, estradiol, insulin-like growth factor-1, thyroid hormones, and bone formation markers in women with FHA (Welt et al., 2004). In a randomized controlled trial lasting 36 weeks, 10 women received metreleptin and 9 women received the placebo. After four weeks, serum total leptin levels in the metreleptin arm increased significantly and continued to rise for the duration of the study. Seven of the ten women receiving metreleptin resumed menstruation and four of those seven were determined to be ovulatory, compared to two of the nine women who resumed menses while receiving placebo. One woman in the metreleptin arm had to withdraw to the study due to the persistent weight loss regardless of the dose adjustments, and one woman became pregnant at week 24 . At the 52-week follow-up, three of the five women who regained menses and completed the entire study continued to experience regular menses (Chou et al., 2011). Long-term administration of leptin has also shown to increase bone mineral density (BMD) and bone mineral content (BMC) by $6 \%$ and $4 \%$ in the lumbar spine compared to baseline values. This two-year pilot study done by Sienkiewicz et al focused on strenuously exercising athletes with FHA and hyperleptinemia. In the first phase lasting 9 months, leptin significantly decreased fat mass leading to dose changes, a common theme in leptin administration that must be carefully monitored. However, $75 \%$ of women resumed menses at 24 months and significantly increased BMD and BMC to reduce the risk of fractures (Sienkiewicz et al., 2011). Adverse effects of metreleptin treatment observed include injection site skin reactions, and persistant weight loss (Chou et al., 2011; Sienkiewicz et al., 2011). More long-term studies examining the therapeutic effects of leptin on FHA are in the process (Chou et al., 2011).

For women who do not wish to conceive at the time of diagnosis and treatment, there are other modalities that can be prescribed to mitigate the consequences of FHA and hypoestrogenism, in particular. Weak estrogen (estriol) administered $2 \mathrm{mg}$ /day orally has been shown to significantly increase LH plasma levels, LH pulse amplitude, and GnRHinduced LH response after 8 weeks in twelve women with FHA (Genazzani et al., 2012). No other hormone parameters were changed throughout the study, and conversion from estriol to estradiol was minimal as seen by the unchanged estradiol plasma concentrations after 8 weeks. Also, none of the women resumed menses (Genazzani et al., 2012). In another study done by Shen, $\mathrm{Xu}$, and Lin at Fudan University in Shanghai, thirty women diagnosed with FHA, 28 of which reported a weight loss of $>10 \%$ prior to amenorrhea, were given estrogen replacement therapy orally at $1 \mathrm{mg} /$ day. Once again, no hormone parameters were altered besides a significant increase in LH plasma levels and GnRH-induced LH response. Nineteen of the thirty women regained menstruation, however, the lack of control subjects makes it difficult to determine whether estrogen facilitated recovery or other confounding factors that were not taken into account (Shen, Xu \& Lin, 2013). In terms of improving BMD and avoiding osteoporosis, there are a number of studies lasting from $1.5-4.3$ years, which report no significant improvements, in addition to no further bone loss, after estrogen therapy in BMD in amenorrheic women compared to age-matched controls (Gibson, Mitchell, Reeve \& Harries, 1999; Karlsson, Weigall, Duan \& Seeman, 2000; Klibanski, Biller, Schoenfeld, Herzog \& Saxe, 1995; Warren, Miller, Olson, Grinspoon \& Friedman, 2005). However, there have been no studies done on the long-term administration of estrogen, which is where the therapeutic value may lay.

The effect of oral contraceptives (OC) on bone health has also been studied in amenorrheic populations. OC differ from estrogen replacement alone by their make up, which can either be progesterone only or a combination of the two with doses that vary widely. From 1990 on, there are seven studies demonstrating positive effects of OC on BMD and nine that saw no effect on BMD. In particular, one study tested lowdose OC (0.03 mg ethinyl estradiol) compared to a placebo in 64 women for 12 months. There was a non-significant increase in BMD, but a significant decrease of $1.2 \%$ in the controls was observed, suggesting that OC are effective in preventing further bone loss rather than increasing BMD (Castelo-Branco, Martinez de Osaba, Pons, \& Vanrell, 1998). On the other hand, a 12-month study comparing OC (0.035 $\mathrm{mg}$ ethinyl estradiol, 0.5-1.0 $\mathrm{mg}$ norethindrone) versus placebo, the OC group had a significant increase in lumbar spine BMD compared to the placebo group (Hergenroeder et al., 1997). There has been no negative effects on BMD reported in women with FHA, therefore, the use of OC to slow the process of bone loss may be a viable option for those who are unable to resume ovulatory menses through other treatment strategies. Altogether, there is a great need for longitudinal studies in the field of pharmaceutical therapies in order to provide the safest and most effective mode of treatment, not only to mitigate the negative impacts amenorrhea has on bone health, but also to reverse anovulation and infertility, as well.

\section{Conclusions \& Discussion}

Hypothalamic amenorrhea is the most common form of secondary amenorrhea that can affect women of all ages and body types. FHA is characterized by suppressed levels of 
circulating gonadotropins and ovarian steroids, due to a weakened pulsatile secretion of GnRH from the hypothalamus. The onset of hypothalamic amenorrhea can be caused by weight loss or chronic reduced energy intake, excessive exercise, or excessive stress. Most often women may present with more than one trigger of onset, due to the interrelated relationship between energy availability and reproductive function. The diminished hypothalamicpituitary-ovarian axis mechanism causes a multitude of neuroendocrine aberrations, including extremely low levels of serum estradiol, low levels of leptin, and elevated levels of cortisol. FHA is considered a reversible condition due to the successful treatment options that aim to resume menstruation and ovulation. However, early diagnosis is very important to avoid long-term complications that include infertility, osteopenia, osteoporosis, and cardiovascular problems. There are many unanswered questions regarding the etiology and pathophysiology of FHA, warranting further research on the mechanisms that cause amenorrhea and the best treatment approaches for women.

First and foremost, the complex regulation of the hypothalamus is not well understood. The link between nutrition, exercise, stress, and reproductive function requires further explanation in order to better understand the origin of this condition. Studies that examine the relative contributions of each factor on reproduction are needed.

Research is required to clarify the role of leptin on the hypothalamus. Although leptin has been shown to induce ovulation and menstruation and positively affect bone health, it is unclear why the administration of human recombinant leptin is not the standard of care for women regardless of their preference to conceive. There is a need more long-term studies conducted in order to verify the safety and efficacy of leptin compared to the administration of gonadotropins or pulsatile GnRH, the current treatment of choice for women who desire to conceive. The effect that leptin has on weight loss must also be examined to provide more accurate dosage, in order to avoid the risk of significant body fat and body weight reduction.

Additionally, more novel treatments aimed to induce menses and mitigate bone loss are ongoing. Research is needed particularly in the areas the aim to reverse stressrelated amenorrhea and hyperactivation of the hypothalamicpituitary-adrenal axis. Psychosocial therapies and hypnotherapy are two natural modes that entail further studies on the long-term efficacy in women following intervention. Pharmaceutical methods that require more research on the effects on women include the administration of opioid antagonists like naltrexone, and administration of gammaaminobutyric acid (GABA), which both play a role in the body's response to stress and anxiety. Furthermore, newer interventions that aim to improve skeletal health must be explored, including administration of insulin-like growth factor 1 and bisphosphonates, which have both shown to slightly increase bone density over time. The potential risks of these treatments are not well known and demand welldesigned studies.

Overall, longitudinal studies are necessary in all areas of treatments in order appreciate the long-term effects of hypothalamic amenorrhea in general. Although FHA is considered a relatively rare condition due to the low prevalence in the entire female population, the health consequences still affect millions of women and most notably the female-athlete population. Education on the effect of weight loss and excessive exercise on menstrual cycles is needed in young women to avoid the associated risks on skeletal and cardiovascular health. Treatment measures should be taken immediately following proper diagnosis to prevent short- and long-term repercussions of this underestimated condition, and an integrated care team including a primary care physical or gynecologist is recommended.

\section{References}

Andrico, S., Gambera, A., Specchia, C., Pellegrini, C., Falsetti, L., \& Sartori, E. (2002). Leptin in functional hypothalamic amenorrhoea. Hum Reprod, 17(8), 2043-2048.

Berga, S. L., Daniels, T. L., \& Giles, D. E. (1997). Women with functional hypothalamic amenorrhea but not other forms of anovulation display amplified cortisol concentrations. Fertil Steril, 67(6), 10241030.

Berga, S. L., Mortola, J. F., Girton, L., Suh, B., Laughlin, G., Pham, P., \& Yen, S. S. (1989). Neuroendocrine aberrations in women with functional hypothalamic amenorrhea. J Clin Endocrinol Metab, 68(2), 301308. doi: 10.1210/jcem-68-2-301

Berga, S.L., Marcus, M.D., Loucks, T.L., Hlastala, S.H., Ringham, R.R., \& Krohn, M.A., (2003). Recovery of ovarian activity in women with functional hypothalamic amenorrhea who were treated with cognitive behavior therapy. Fertil Steril 80(4), 97681.

Bomba, M., Gambera, A., Bonini, L., Peroni, M., Neri, F., Scagliola, P., \& Nacinovich, R. (2007). Endocrine profiles and neuropsychologic correlates of functional hypothalamic amenorrhea in adolescents. Fertil Steril, 87(4), 876-885. doi: 10.1016/j.fertnstert.2006.09.011

Bonen, A. (1994). Exercise-induced menstrual cycle changes. A functional, temporary adaptation to metabolic stress. Sports Med, 17(6), 373-392.

Bullen, B. A., Skrinar, G. S., Beitins, I. Z., von Mering, G., Turnbull, B. A., \& McArthur, J. W. (1985). Induction of menstrual disorders by strenuous exercise in untrained women. $N$ Engl $J$ Med, 312(21), 1349-1353. doi: 10.1056/NEJM198505233122103

Cameron, J. L., Helmreich, D. L., \& Schreihofer, D. A. (1993). Modulation of reproductive hormone secretion by nutritional intake: stress signals versus metabolic signals. Hum Reprod, 8 Suppl 2, 162-167.

Caronia, L. M., Martin, C., Welt, C. K., Sykiotis, G. P., Quinton, R., Thambundit, A., . . . Pitteloud, N. (2011). A genetic basis for functional hypothalamic amenorrhea. $N$ Engl J Med, 364(3), 215-225. doi: 10.1056/NEJMoa0911064

Castelo-Branco, C., Martinez de Osaba, M. J., Pons, F., \& Vanrell, J. A. (1998). Effects on bone mass of two oral contraceptives containing ethinylestradiol and cyproterone acetate or desogestrel: results of a 2- 
year follow-up. Eur J Contracept Reprod Health Care, 3(2), 79-84.

Chou, S. H., Chamberland, J. P., Liu, X., Matarese, G., Gao, C., Stefanakis, R., Mantzoros, C. S. (2011). Leptin is an effective treatment for hypothalamic amenorrhea. Proc Natl Acad Sci U S A, 108(16), 6585-6590. doi: 10.1073/pnas.1015674108

De Souza, M. J., Leidy, H. J., O'Donnell, E., Lasley, B., \& Williams, N. I. (2004). Fasting ghrelin levels in physically active women: relationship with menstrual disturbances and metabolic hormones. $J$ Clin Endocrinol Metab, 89(7), 3536-3542. doi: 10.1210/jc.2003-032007

De Souza, M. J., \& Williams, N. I. (2005). Beyond hypoestrogenism in amenorrheic athletes: energy deficiency as a contributing factor for bone loss. Curr Sports Med Rep, 4(1), 38-44.

Dhillo,W.S., Chaudhri, O.B., Thompson E.L., Murphy, K.G., Patterson, M., ... Bloom, S.R. (2007). Kisspeptin54 stimulates gonadotropin release most potently during the preovulatory phase of the menstrual cycle in women. J Clin Endocrinol Metab, 92(10), 3958-66.

Dundon, C. M., Rellini, A. H., Tonani, S., Santamaria, V., \& Nappi, R. (2010). Mood disorders and sexual functioning in women with functional hypothalamic amenorrhea. Fertil Steril, 94(6), 2239-2243. doi: 10.1016/j.fertnstert.2010.01.012

Facchinetti, F., Fava, M., Fioroni, L., Genazzani, A. D., \& Genazzani, A. R. (1993). Stressful life events and affective disorders inhibit pulsatile LH secretion in hypothalamic amenorrhea. Psychoneuroendocrinology, 18(5-6), 397-404.

Furuta, M., Funabashi, T., \& Kimura, F. (2001). Intracerebroventricular administration of ghrelin rapidly suppresses pulsatile luteinizing hormone secretion in ovariectomized rats. Biochem Biophys Res Commun, 288(4), 780-785. doi: $10.1006 /$ bbrc. 2001.5854

Genazzani, A. D. (2005). Neuroendocrine aspects of amenorrhea related to stress. Pediatr Endocrinol Rev, 2(4), 661-668.

Genazzani, A. D., Meczekalski, B., Podfigurna-Stopa, A., Santagni, S., Rattighieri, E., Ricchieri, F., . . . Simoncini, T. (2012). Estriol administration modulates luteinizing hormone secretion in women with functional hypothalamic amenorrhea. Fertil Steril, 97(2), 483-488. doi: 10.1016/j.fertnstert.2011.11.004

Genazzani, A. D., Petraglia, F., Fabbri, G., Monzani, A., Montanini, V., \& Genazzani, A. R. (1990). Evidence of luteinizing hormone secretion in hypothalamic amenorrhea associated with weight loss. Fertil Steril, 54(2), 222-226.

Genazzani, A. D., Ricchieri, F., Lanzoni, C., Strucchi, C., \& Jasonni, V. M. (2006). Diagnostic and therapeutic approach to hypothalamic amenorrhea. Ann $N Y$ Acad Sci, 1092, 103-113. doi: 10.1196/annals.1365.009

Gibson, J. H., Mitchell, A., Reeve, J., \& Harries, M. G.
(1999). Treatment of reduced bone mineral density in athletic amenorrhea: a pilot study. Osteoporos Int, 10(4), 284-289. doi: 10.1007/s001980050228

Giles, D. E., \& Berga, S. L. (1993). Cognitive and psychiatric correlates of functional hypothalamic amenorrhea: a controlled comparison. Fertil Steril, 60(3), 486-492.

Golden, N.H., \& Carlson, J.L., (2008). The Pathophysiology of Amenorrhea in the Adolescent. Ann N Y Acad Sci, 1135(1) 163-78. doi: 10.1196/annals.1429.014

Golden, N.H., Jacobson, M.S., Sterling, W.M., \& Hertz, S. (2008). Treatment Goal Weight in Adolescents with Anorexia Nervosa: Use of BMI Percentiles. Int J Eat Disord, 41(4), 301-306.

Golden, N. H., \& Shenker, I. R. (1994). Amenorrhea in anorexia nervosa. Neuroendocrine control of hypothalamic dysfunction. Int J Eat Disord, 16(1), 53-60.

Gordon, C. M. (2010). Clinical practice. Functional hypothalamic amenorrhea. N Engl J Med, 363(4), 365-371. doi: 10.1056/NEJMcp0912024

Hergenroeder, A. C., Smith, E. O., Shypailo, R., Jones, L. A., Klish, W. J., \& Ellis, K. (1997). Bone mineral changes in young women with hypothalamic amenorrhea treated with oral contraceptives, medroxyprogesterone, or placebo over 12 months. Am J Obstet Gynecol, 176(5), 1017-1025.

Hind, K. (2008). Recovery of bone mineral density and fertility in a former amenorrheic athlete. J Sports Sci Med, 7(3), 415-418.

Jayasena, C.N., Abbara, A., Veldhuis, J.D., Comninos, A.N., Ratnasabapathy, R., De Silva, A., ... Dhillo, W.S. (2014). Increasing LH Pulsatility in Women with Hypothalamic Amenorrhea Using Intravenous Infusion of Kisspeptin-54. J Clin Endocrinol Metab, 99(6), 953-61. doi: 10.1210/jc.2013-1569

Jayasena, C. N., Nijher, G. M., Abbara, A., Murphy, K. G., Lim, A., Patel, D., . . Dhillo, W. S. (2010). Twiceweekly administration of kisspeptin-54 for 8 weeks stimulates release of reproductive hormones in women with hypothalamic amenorrhea. Clin Pharmacol Ther, 88(6), 840-847. doi: 10.1038/clpt.2010.204

Kaplan, J. R., \& Manuck, S. B. (2008). Ovarian dysfunction and the premenopausal origins of coronary heart disease. Menopause, 15(4 Pt 1), 768-776.

Karlsson, M. K., Weigall, S. J., Duan, Y., \& Seeman, E. (2000). Bone size and volumetric density in women with anorexia nervosa receiving estrogen replacement therapy and in women recovered from anorexia nervosa. J Clin Endocrinol Metab, 85(9), 3177-3182. doi: 10.1210/jcem.85.9.6796

Kolaczynski, J. W., Ohannesian, J. P., Considine, R. V., Marco, C. C., \& Caro, J. F. (1996). Response of leptin to short-term and prolonged overfeeding in humans. J Clin Endocrinol Metab, 81(11), 41624165. doi: 10.1210/jcem.81.11.8923877

Kopp-Woodroffe, S. A., Manore, M. M., Dueck, C. A., Skinner, J. S., \& Matt, K. S. (1999). Energy and nutrient status of amenorrheic athletes participating in a diet and exercise training intervention program. Int J Sport Nutr, 9(1), 70-88.

Lawson, E. A., Donoho, D., Miller, K. K., Misra, M., 
Meenaghan, E., Lydecker, J., . . . Klibanski, A. (2009). Hypercortisolemia is associated with severity of bone loss and depression in hypothalamic amenorrhea and anorexia nervosa. $J$ Clin Endocrinol Metab, 94(12), 4710-4716. doi: 10.1210/jc. 2009-1046

Liu, J. H., \& Bill, A. H. (2008). Stress-associated or functional hypothalamic amenorrhea in the adolescent. Ann N Y Acad Sci, 1135, 179-184. doi: 10.1196/annals.1429.027

Loucks, A.B., Verdun, M., \& Heath, E.M., (1998). Low energy availability, not stress of exercise, alters LH Pulsatility in exercising women. J Appl Physiol, 84(1), 37-46.

Macut, D., Micic, D., Pralong, F. P., Bischof, P., \& Campana, A. (1998). Is there a role for leptin in human reproduction? Gynecol Endocrinol, 12(5), 321-326.

Marcus, M. D., Loucks, T. L., \& Berga, S. L. (2001). Psychological correlates of functional hypothalamic amenorrhea. Fertil Steril, 76(2), 310-316.

Marshall, L. A. (1994). Clinical evaluation of amenorrhea in active and athletic women. Clin Sports Med, 13(2), 371-387.

Martin, K.A., Hall, J.E., Adams, J.M., \& Crowley, W.F. (1993). Comparison of Exogenous Gonadotropins and Pulsatile Gonadotropin-Releasing Hormone for Induction of Ovulation in Hypogonadotropic Amenorrhea. J Clin Endocrinol Metab 77(1), 125-9. doi: 10.1210/jcem.77.1.8325934.

Meczekalski, B., Katulski, K., Czyzyk, A., Podfigurna-Stopa, A., \& Maciejewska-Jeske, M. (2014). Functional hypothalamic amenorrhea and its influence on women's health. $J$ Endocrinol Invest. doi: 10.1007/s40618-014-0169-3

Meczekalski, B., Podfigurna-Stopa, A., WarenikSzymankiewicz, A., \& Genazzani, A. R. (2008). Functional hypothalamic amenorrhea: current view on neuroendocrine aberrations. Gynecol Endocrinol, 24(1), 4-11. doi: 10.1080/09513590701807381

Mendelsohn, M.E., \& Karas, R.H., (1994). Estrogen and the blood vessel wall. Curr Opin Cardiol 9(5), 619-26

Mendelsohn, M. E., \& Karas, R. H. (1999) The Protective Effects of Estrogen on the Cardiovascular System. N Engl J Med, 340(23), 1801-11.

Michopoulos, V., Mancini, F., Loucks, T. L., \& Berga, S. L. (2013). Neuroendocrine recovery initiated by cognitive behavioral therapy in women with functional hypothalamic amenorrhea: a randomized, controlled trial. Fertil Steril, 99(7), 2084-2091 e2081. doi: 10.1016/j.fertnstert.2013.02.036

Nattiv, A., Loucks, A. B., Manore, M. M., Sanborn, C. F., Sundgot-Borgen, J., Warren, M. P., \& American College of Sports, M. (2007). American College of Sports Medicine position stand. The female athlete triad. Med Sci Sports Exerc, 39(10), 1867-1882. doi: 10.1249/mss.0b013e318149f111

Shen, Z. Q., Xu, J. J., \& Lin, J. F. (2013). Resumption of menstruation and pituitary response to gonadotropin-releasing hormone in functional hypothalamic amenorrhea subjects undertaking estrogen replacement therapy. J Endocrinol Invest, 36(10), 812-815. doi: 10.3275/8939

Sienkiewicz, E., Magkos, F., Aronis, K. N., Brinkoetter, M., Chamberland, J. P., Chou, S., . . . Mantzoros, C. S. (2011). Long-term metreleptin treatment increases bone mineral density and content at the lumbar spine of lean hypoleptinemic women. Metabolism, 60(9), 1211-1221. doi: 10.1016/j.metabol.2011.05.016

Snyder, P. J., Reitano, J. F., \& Utiger, R. D. (1975). Serum LH and FSH responses to synthetic gonadotropinreleasing hormone in normal men. $J$ Clin Endocrinol Metab, 41(5), 938-945. doi: 10.1210/jcem-41-5-938

Tschugguel, W., \& Berga, S. L. (2003). Treatment of functional hypothalamic amenorrhea with hypnotherapy. Fertil Steril, 80(4), 982-985.

Warren, M. P., Miller, K. K., Olson, W. H., Grinspoon, S. K., \& Friedman, A. J. (2005). Effects of an oral contraceptive (norgestimate/ethinyl estradiol) on bone mineral density in women with hypothalamic amenorrhea and osteopenia: an open-label extension of a double-blind, placebo-controlled study. Contraception, 72(3), 206-211. doi: 10.1016/j.contraception.2005.03.007

Welt, C. K., Chan, J. L., Bullen, J., Murphy, R., Smith, P., DePaoli, A. M., . . . Mantzoros, C. S. (2004). Recombinant human leptin in women with hypothalamic amenorrhea. N Engl J Med, 351(10), 987-997. doi: 10.1056/NEJMoa040388

Wiksten-Almstromer, M., Hirschberg, A. L., \& Hagenfeldt, K. (2007). Menstrual disorders and associated factors among adolescent girls visiting a youth clinic. Acta Obstet Gynecol Scand, 86(1), 65-72. doi: 10.1080/00016340601034970 